\title{
Le projet Aramis et la prévision à courte échéance
}

\section{The Aramis project and short-range forecasting}

\author{
M. Gilet et M. Ciccione \\ Direction de la Météorologie \\ Etablissement d'Etudes et de Recherches Météorologiques \\ Centre de Recherche en Physique de l'Atmosphère \\ 78470 Magny-les-Hameaux
}

\section{Objectifs du projet}

Le radar météorologique constitue un moyen privilégié pour détecter les phénomènes dangereux et pour évaluer, de façon quasi instantanée, la répartition des intensités de précipitations sur des surfaces étendues.

Les études faites à l'étranger et les résultats de l'expérience HYDROMEL, conduite dans la région parisienne, ont abondamment démontré que dans des conditions convenables d'exploitation la qualité des mesures radar était suffisante pour satisfaire une large gamme de besoins en météorologie et en hydrologie (Froment, 1979, Wilson \& Brandes, 1979).

Le développement récent des possibilités de traitement et de transmission de l'information permet par ailleurs de diffuser, largement et à faible coût, les cartes de précipitations obtenues par les radars météorologiques. Par rapport au réseau météorologique conventionnel, le radar présente notamment les avantages suivants :

- une disponibilité immédiate de l'information,

- une fréquence de renouvellement des données de l'ordre de quelques minutes contre quelques heures pour le réseau actuel,

- une résolution spatiale de l'ordre du kilomètre, avec des données naturellement lissées. Le réseau pluviométrique a, au mieux, quelques dizaines de kilomètres de résolution et les mesures sont de nature ponctuelle, ce qui peut laisser échapper certains phénomènes (grains, orages),

- une précision dans les mesures de lame d'eau intégrées dans l'espace et le temps qui peut être considérée comme comparable, sinon supérieure, à ce que fournit actuellement le réseau de stations de la Météorologie, dans la mesure où on souhaite obtenir des réponses en temps quasi réel.

Ces avantages justifient l'utilisation de radars pour une meilleure appréciation du temps présent, à petite et moyenne échelles: de quelques kilomètres à 1000 kilo- mètres dans l'espace, de quelques minutes à quelques heures dans le temps. Cette connaissance très fine de la répartition des précipitations est nécessaire pour obtenir des prévisions précises à quelques heures d'échéance, dans un domaine où les modèles numériques de simulation de l'atmosphère sont actuellement inutilisables.

Le radar a certes été utilisé depuis ses débuts par les météorologistes, tout autant pour des objectifs opérationnels que dans des buts de recherches en physique des nuages. En France, plusieurs grands aéroports bénéficient ainsi depuis longtemps d'une surveillance radar pour l'alerte aux orages. Peu à peu, de nombreux usagers autres que la navigation aérienne, tels les réseaux d'assainissement urbains, les agriculteurs, les sociétés d'exploitation d'autoroutes, ont pris l'habitude de demander régulièrement des renseignements aux stations radar de la Météorologie.

Les informations sont fournies par les personnels exploitant le radar, soit sous forme de messages standard envoyés par voie télégraphique pendant les périodes d'alerte (RADOB, RASIG), soit par téléphone. Ceci demande souvent une analyse spécifique de l'image disponible sur le scope radar et donc du temps aux personnels. L'immense majorité des stations du réseau météorologique n'ont aucun accès à l'information radar, ce qui les met dans une situation paradoxale à laquelle le grand public est malheureusement habitué, pour ce qui concerne la connaissance du temps présent.

Un des tous premiers objectifs du projet ARAMIS est de permettre à l'ensemble des stations météorologiques départementales d'avoir un accès facile aux informations radar et satellite. La manière la plus économique d'y parvenir est de concentrer les images en utilisant des lignes téléphoniques spécialisées déjà existantes. Cette concentration des données et la représentation des images, radar et satellite, sur une aire englobant la France, permettra par ailleurs de progresser dans les trois domaines suivants :

- la compréhension globale de chaque situation concrète grâce à la comparaison des données fournies par

LA HOUILLE BLANCHE/N $N^{\circ} 5 / 6-1983$ 
les radars et les satellites avec les autres données disponibles; celles-ci comportent en particulier les observations au sol et en altitude, ainsi que les résultats des modèles numériques à maille fine (modèle PERIDOT actuellement en cours de développement à la Météorologie).

- la prévision de la répartition des précipitations pour des échéances allant de une à six heures. Dans un premier temps, ces prévisions seront faites à partir des données radar seules. Les incertitudes seront encore importantes, mais seront considérablement réduites par rapport à ce qui peut être obtenu par tout autre moyen. A plus long terme, on peut espérer que l'intégration d'autres données au processus de prévision à très courte échéance conduira à des améliorations sensibles, et qu'il sera possible d'effectuer une jonction avec les prévisions foumies par les modèles numériques, dont la validité ne débute que six à douze heures après l'instant de la mesure (latence due à la collecte des données, temps de calcul et de mise en équilibre du modèle). - la climatologie des précipitations, dont l'élaboration demande actuellement des moyens humains importants. et un long délai de dépouillement. Une utilisation intensive et soigneuse des radars dans ce domaine permettra d'alléger le travail des climatologistes et des observateurs, d'améliorer les procédures de validation des données, et de réduire le temps d'édition de certains documents foumis aux usagers.

Les trois utilisations énumérées ci-dessus exigent qu'on. dispose de cartes couvrant la France et renouvelées fréquemment. La portée utile d'un radar météorologique dépasse rarement 150 kilomètres, ce qui est insuffisant au regard des dimensions caractérisant l'organisation de nombreux phénomènes atmosphériques (plusieurs centaines de kilomètres).

Par ailleurs, les délais d'alerte obtenus avec un seul radar sont courts, les vitesses de déplacement des cellules pluvieuses dépassant fréquemment $100 \mathrm{~km} / \mathrm{h}$ sous nos latitudes.

La première priorité du projet consiste donc à réaliser cette mosaïque radar, qui sera renouvelée toutes les quinze minutes, et à diffuser cette information.

Les estimations pluviométriques et les prévisions à courte échéance, obtenues à l'aide du réseau radar, permettront à la Météorologie d'améliorer notablement la qualité des prestations qu'elle fournit déjà à de nombreux usagers, tels les services d'annonce de crues, la protection civile, l'E.D.F., l'aéronautique, le réseau routier ou les particuliers.

La précision de la mesure de la lame d'eau sur environ une heure pour une zone d'au moins $100 \mathrm{~km}^{2}$ convenablement couverte par un radar sera de l'ordre de $50 \%$ en erreur relative moyenne, à raison de 4 à 6 balayages horaires du radar. Cette erreur pourra être réduite en accroissant le domaine spatial considéré et la période temporelle d'intégration. Ceci est tout à fait suffisant pour de nombreuses applications.

Cependant, certains usagers souhaiteront disposer soit de résultats plus précis, soit de données spécialement élaborées à leur intention. Il a été démontré, qu'en utilisant de manière conjointe un radar et quelques pluviographes servant de vérité sol, l'erreur moyenne sur la mesure de lame d'eau horaire dans de bonnes condi- tions d'exploitation peut être réduite à moins de $25 \%$. Des mesures précises de cette nature ne pourront être conduites de façon opérationnelle qu'en liaison étroite avec les utilisateurs directs, en effectuant un partage de tâches adapté aux moyens et aux désirs clairement exprimés par les parties en présence.

Dans un premier temps, l'estimation de lames d'eau à $25 \%$ près n'apparaît pas en première priorité dans le calendrier de réalisation du projet ARAMIS, la Météorologie considérant que l'effort doit être porté au départ sur la constitution du réseau, et que l'estimationn approximative des intensités de pluie suffira à aborder les trois objectifs énoncés plus haut et à satisfaire de nombreux usagers. Cependant, des actions ponctuelles pourront être conduites concernant l'hydrologie précise, sous la forme de projets pilotes, dans la mesure où elles ne gêneront pas la réalisation du réseau.

\section{Les trois phases de la réalisation}

Les objectifs énoncés ci-dessus seront atteints progressivement, au cours des trois phases du projet, qui s'éten. dra sur cinq ans. On trouvera ci-dessous les principales réalisations prévues pour chacune de ces trois phases dans les quatre domaines suivants d'activité :

- les radars

- la centralisation

- la diffusion

- la recherche

On abordera ensuite la description plus détaillée des moyens existants et des projets en cours dans ces différents domaines.

Phase I - aboutissement prévu avant fin 1984 des aménagements suivants :

- numérisation des radars MELODI de Dammartin, Brest et Bordeaux,

- numérisation du radar du Service Hydrologique Centralisateur de Perigueux (radar de Grèzes),

- installation de radars RODIN à Toulouse, Nancy et Nantes,

- mise en place des moyens nécessaires pour que cinq radars au moins (Dammartin, Bordeaux, Brest, Marseille, Lyon) transmettent un PPI toutes les 15 minutes vers Paris,

- concentration sur le calculateur CDC 835 de Paris des données d'au moins cinq radars (Dammartin, Bordeaux, Brest, Marseille, Lyon) et des données du réseau britannique,

- réalisation d'une mosaïque radar toutes les 15 minutes, en juxtaposant les données brutes des radars, et diffusion de cette mosaïque sur des lignes alimentant les stations météorologiques (terminaux METEOTEL), - mise en ouvre d'un archivage des données radar et satellite à Paris,

- diffusion depuis Paris sur les lignes alimentant les stations METEOTEL d'images METEOSAT traitées au Centre de Météorologie Spatiale de Lannion,

- industrialisation et mise au point des terminaux de réception d'images METEOTEL,

- installation de METEOTEL dans une trentaine de stations météorologiques, 


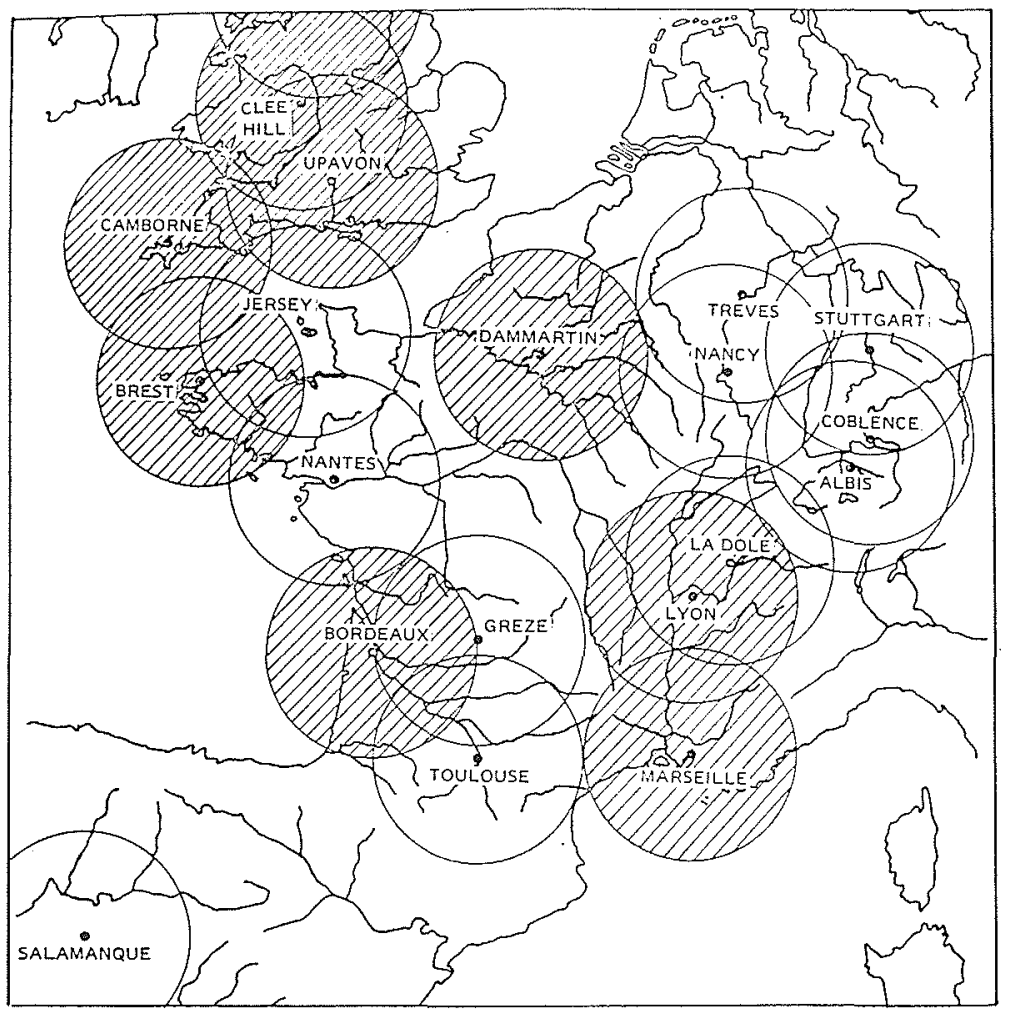

Figure 1 - IMAGE METEOTEL - Centrée sur $2^{\circ}$ LONG E - $47^{\circ}$ LAT N. Projection stréréographique polaire. Portée théorique des radars $150 \mathrm{~km}$. Zone hachurée : radars disponibles fin 84.

- mise au point de la méthode de prévision de la pluie à courte échéance à partir des données radar seules qui sera opérationnelle en phase II,

- début de constitution d'un système interactif expérimental pour le développement des méthodes de traitement et d'analyse simultanée des données radar et satellite,

- étude des améliorations les plus urgentes à apporter aux systèmes radar,

- participation à une expérience française sur les fronts en collaboration avec le Centre National de Recherches Météorologiques de Toulouse,

Phase II - Mise en service avant fin 1986 des opérations ci-après :

- automatisation des radars de Nancy, Grèzes, et Toulouse,

- déplacement vers un autre site d'un radar RODIN actuellement implanté au voisinage d'Agen, pour une expérience temporaire,

- amélioration de la couverture de la région parisienne (suppression des échos de sol du radar de Dammartin ou autre solution),

- concentration sur Paris des données de tous les radars français portés sur la figure 1, ainsi que de quelques radars étrangers si cela s'avère possible,

- mise en place sur quelques radars des moyens permettant la mesure de la lame d'eau en relation avec des utilisateurs de ce type de données,

- mise en place à Paris d'une procédure systématique de vérification et d'analyse des données radar et satellite, - réalisation opérationnelle et diffusion de prévisions de pluie à très courte échéance en utilisant uniquement les données radar,

- équipement de toutes les stations du réseau météorologique français en terminaux METEOTEL,

- possibilité d'accéder aux données radar centralisées ou à des informations plus détaillées provenant directement des radars en utilisant le réseau téléphonique commuté,

- étude de définition d'un nouveau modèle de radar météorologique adapté aux besoins d'ARAMIS (radar de cinquième génération),

- étude des possibilités d'utilisation simultanée des données radar/satellite, des autres données du réseau météorologique et des résultats des modèles numériques pour l'amélioration des prévisions à courte échéance, - définition d'un système opérationnel intégré pour la prévision à courte échéance.

Phase III - Fin du programme à fin 1987 avec :

- construction et essai du prototype du radar météorologique de cinquième génération adapté aux besoins d'ARAMIS,

- mise en ouvre du système opérationnel intégré de prévision à courte échéance,

- intensification des échanges de données au niveau européen.

\section{Les sources de données}

Les radars, actuellement existants en France, qui seront utilisés pour le projet ARAMIS sont au nombre de dix. Trois de ces radars sont des $10 \mathrm{~cm}$ de type 
MELODI et six des $5 \mathrm{~cm}$ de type RODIN. Le dixième radar est un $5 \mathrm{~cm}$ appartenant au Service Hydrologique Centralisateur de Périgueux. Il existe un onzième radar $5 \mathrm{~cm}$, de type RODIN, situé à Trappes près de Paris; ce radar sert actuellement à la maintenance et à la formation des personnels. Les principales caractéristiques des MELODI et des RODIN sont indiquées dans le Tableau I.

Tableau I

Caractéristiques des radars MELODI et RODIN

\begin{tabular}{|l|c|c|}
\hline & MELODI & RODIN \\
\hline $\begin{array}{l}\text { Constructeur } \\
\text { Longueur d'onde } \\
\text { (cm) } \\
\text { Fréquence de } \\
\text { recurrence (Hz) } \\
\text { Durée de l'impulsion } \\
\text { ( } \mu \text { s) }\end{array}$ & OMERA & THOMSON CSF \\
$\begin{array}{c}\text { Puissance émise (KW) } \\
\text { Diamètre de l'aérien } \\
\text { (m) }\end{array}$ & 10,7 & 5,3 \\
$\begin{array}{c}\text { Largueur du faisceau } \\
\text { (degrés) } \\
\text { Signal minimum } \\
\text { détectable (dBm) } \\
\text { Système de numéri- } \\
\text { sation } \\
\text { Portée utilisée pour } \\
\text { la centralisation } \\
\text { (km) }\end{array}$ & 100 & 330 \\
\hline
\end{tabular}

Les radars RODIN sont d'acquisition récente. Ils sont équipés, à la livraison, d'un système numérique de traitement des images, le MT 750. Ce matériel permet de visualiser l'image sur un écran couleur et de la transmettre par ligne téléphonique. Il est possible, par ailleurs, d'effectuer de nombreuses manipulations sur l'image comme des changements d'échelles, zooms ou excentrements. Le MT 750 à été programmé au Centre
Technique et du Matériel de la Météorologie pour contrôler les mouvements de l'antenne.

Les radars MELODI sont des matériels plus anciens, non numérisés à l'origine. Cette numérisation a été réalisée à la Météorologie, à l'aide d'un système baptisé SAPHYR. Ce matériel permet de visualiser l'image sur un écran couleur et de la transmettre par ligne téléphonique. On peut également procéder à une variété de manipulations d'image, de façon sensiblement équivalente au MT750. SAPHYR contrôle les mouvements de l'antenne et peut recevoir un système d'enregistrement sur bandes magnétiques.

La figure 1 donne les emplacements des radars français, hormis un radar RODIN dont le site n'est pas définitif. Certains radars européens dont les données pourraient avoir un intérêt pour le réseau français figurent également sur la carte. La zone géographique couverte par cette figure correspond à la superficie de la mosaïque radar qui sera réalisée à Paris. Les trois radars MELODI sont ceux de Dammartin en Goëlle, Brest et Bordeaux. Les deux derniers sites sont très favorables à l'implantation d'une station radar. A Dammartin en Goëlle, on observe des échos de sol sur plus de cinquante kilomètres dans la plupart des directions; une étude est en cours pour essayer de remédier à cet inconvénient. Trois radars de type RODIN sont installés à Marseille, Lyon et Toulouse. Les deux premiers sont actuellement masqués dans certaines directions, en raison des montagnes avoisinantes; dans leurs cas, les portées indiquées sur la figure 1 sont donc peu réalistes. Deux autres radars RODIN seront installés prochainement à Nancy et à Nantes. Le site définitif d'un radar RODIN qui se trouve actuellement à titre temporaire dans la région d'Agen, pour une expérience sur la grêle, n'est pas encore fixé. A la suite d'un accord avec la Direction Départementale de l'Equipement de Périgueux, la Météorologie aura accès aux données transmises par un radar $5 \mathrm{~cm}$ situé à Grèzes et appartenant au Service Hydrologique Centraliseur de Périgueux. Enfin, on peut constater que les

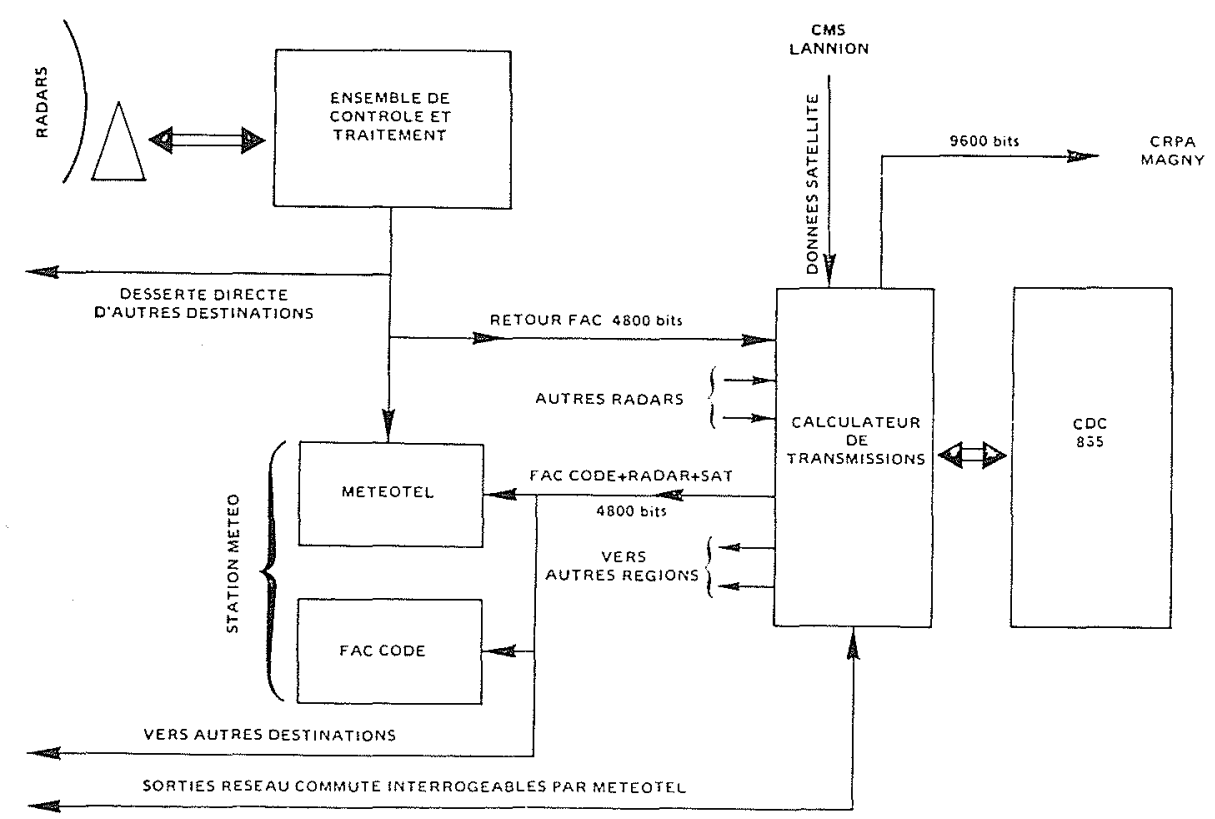

Figure 2 - Principes retenus pour la concentration et la rediffusion des données satellite et radar. 


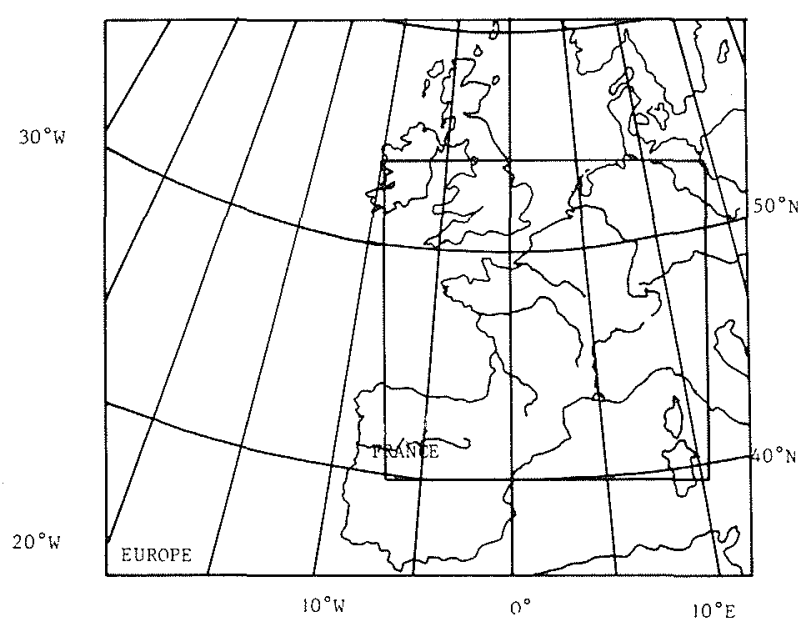

Figure 3 - Formats METEOTEL.

données en provenance de radars existant actuellement dans des pays comme la Grande-Bretagne, la RFA ou la Suisse seraient d'un grand intérêt pour prévoir plus à l'avance l'arrivée de précipitations.

Pendant les premières années, les modes de balayage des radars seront simplifiés au maximum. Chaque radar devra pouvoir transmettre au moins un tour d'horizon à site bas toutes les 15 minutes. Ces données iront vers Paris et vers d'autres utilisateurs éventuels en passant dans la quasi totalité des cas par la station météorologique la plus proche et par le Centre Météorologique Régional (Fig. 2). L'intervalle de 15 minutes est trop long pour de nombreuses applications, comme par exemple la mesure précise de hauteur de pluie sur de petites surfaces. (Wilson et Brandes, 1979). Les radars appelés à faire ce type de mesure pourront balayer plus fréquemment. De même, il pourra être envisagé à terme pour certains radars d'effectuer des balayages à plusieurs sites et de transmettre des données à altitude constante (CAPPI) (Constant Altitude Plane Position Indicator) si cela se justifie, dans le but de réduire des échos de sol par exemple.

Les modes d'opération initiaux seront simples pour éviter, dès le début, de modifier les systèmes MT 750 et SAPHYR. Ces systèmes sont suffisants pour au moins la première phase du projet, où on n'exigera pas une fiabilité parfaite des installations. Cependant, ils demanderont à ètre renforcés sinon remplacés assez rapidement afin qu'on puisse disposer en particulier des possibilités suivantes:

- fonctionnement entièrement automatique de certains radars comme celui de Nancy, où il ne sera pas possible de maintenir du personnel en permanence,

- amélioration des traitements de données sur place, pour l'élimination des échos fixes, ou pour des mesures hydrologiques par exemple,

- augmentation des possibilités de transmission vers différents types d'usagers.

On projette d'effectuer à la Météorologie les développements nécessaires pour équiper les radars existants dans ce sens. Les radars supplémentaires qui pourront être acquis ultérieurement, devront autant que possible être conçus pour accepter facilement les fonctions nécessaires à un mode d'opération optimal du réseau.

Les données satellitaires utilisées pour le projet ARAMIS seront celles de METEOSAT, en visible et en infra-rouge, ou éventuellement celles des satellites de la série NOAA si METEOSAT venait à faire défaut. Ces données sont déjà traitées au Centre de Météorologie Spatiale de Lannion en Bretagne et seront transmises vers Paris sous deux formats superposables à celui des données radar (Fig. 3). Le premier, dit format METEOTEL France, aura une résolution d'environ $6 \times 6 \mathrm{~km}^{2}$. Le second, dit format METEOTEL Europe aura une résolution de $12 \times 12 \mathrm{~km}^{2}$. La projection utilisée est la projection stéréographique polaire.

$\mathrm{Au}$ cours de la première phase du projet, l'utilisation conjointe des images satellite et radar sera relativement

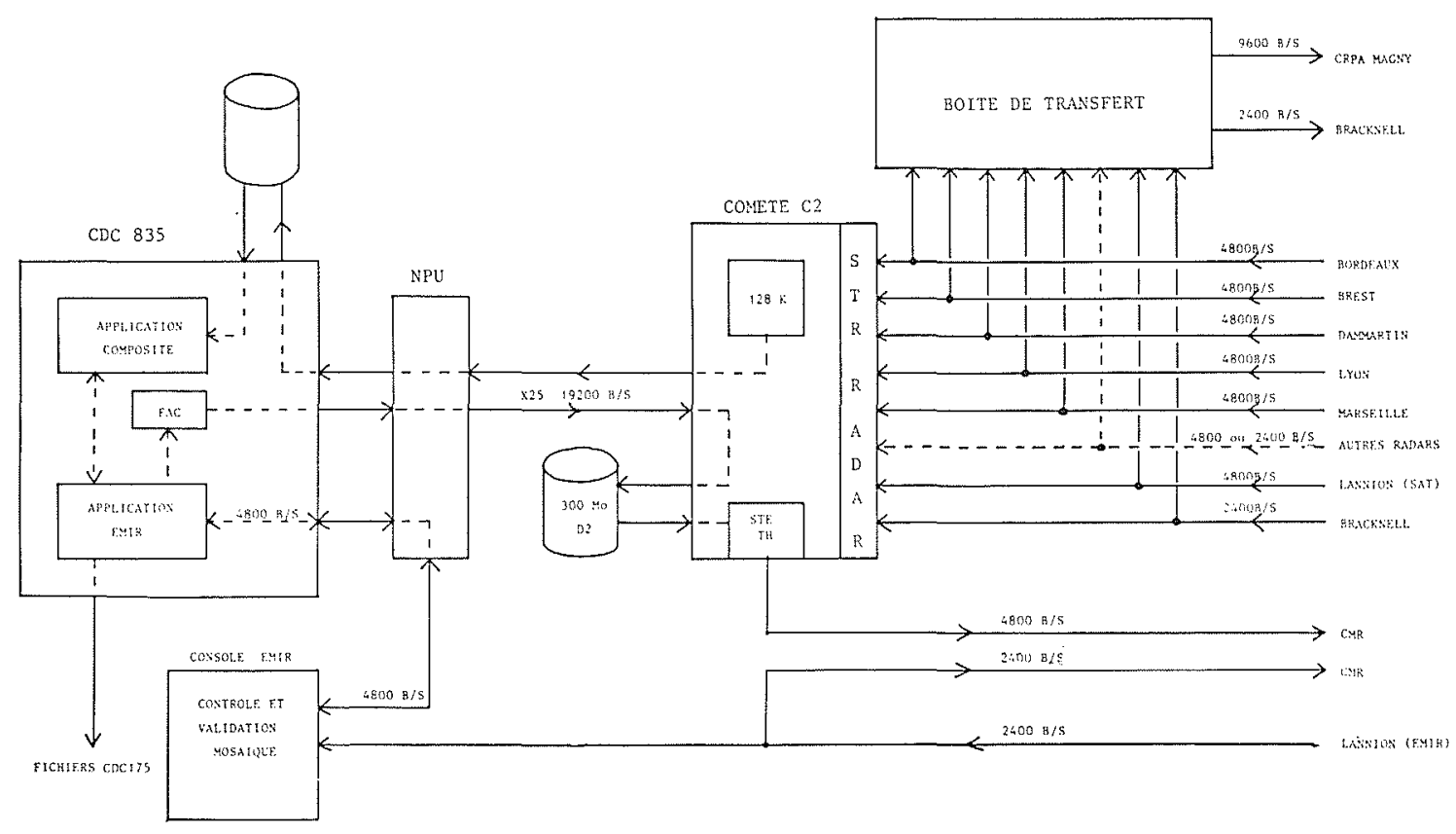

Figure 4 - Concentration des données RADAR et METEOSAT - Schéma général. 
limitée et conduite manuellement. Les méthodes de traitement automatique simultané de ces deux types d'images ne pourront être mises au point que progressivement et ne passeront probablement pas à un stade opérationnel avant le début de la phase III.

\section{Centralisa tion des données}

Les données radar et METEOSAT seront transmises vers Paris en utilisant la plupart du temps des canaux existants, qui correspondent aux "voies retour" des lignes téléphoniques spécialisées alimentant les stations météorologiques en cartes sur des récepteurs fac similé. $\mathrm{Au}$ cours de la phase I et probablement de toute la phase II du projet, la centralisation opérationnelle des données sera réalisée avec des moyens informatiques dont la grande majorité existe déjà. Parallèlement, un système expérimental sera mis sur pied en utilisant des moyens distincts de mini et micro-informatique, de manière à ce que les développements n'interfèrent pas avec un bon fonctionnement du réseau. Les principaux moyens utilisés pour la concentration opérationnelle, selon le principe de la figure 4 , sont les suivants :

1) Un minicalculateur MITRA 225 baptisé COMETE2, chargé de gérer l'ensemble des télécommunications, recevra les données de chacun des radars, de METEOSAT en provenance du Centre de Météorologie Spatiale de Lannion et du réseau britannique transmises depuis Bracknell. La vitesse de transmissions sera le plus souvent de $4800 \mathrm{~b} / \mathrm{s}$, ce qui correspond à un temps d'une minute environ pour une image radar. Certaines lignes fonctionneront à $2400 \mathrm{~b} / \mathrm{s}$. Le calculateur MITRA n'effectuera aucun traitement sur les données, il se contentera de les renvoyer par une liaison rapide en mode $X 25$ vers le calculateur CDC 835 . Après traitement des données sur ce calculateur, les mosaïques radar et les images satellitaires seront retoumées à COMETE2, qui les transmettra aux stations du réseau en utilisant les lignes spécialisées à $4800 \mathrm{~b} / \mathrm{s}$ servant à la diffusion des cartes météorologiques.

2) Deux calculateurs CDC 835 et CDC 175, qui constituent actuellement l'essentiel des moyens informatiques de la Météorologie à Paris. Le CDC 835, connecté directement à COMETE effectuera la gestion routinière des données, constituera la mosaïque radar et fera les calculs nécessaires aux prévisions. Le calculateur sera en particulier relié à une console graphique EMIR. Le calculateur CYBER 175 sera utilisé pour les développements, la mise au point des programmes et l'archivage des données. Il servira également de secours du CDC 835.

3) Une console graphique EMIR, reliée au calculateur CDC 835 par une liaison série, contrôlera la qualité des images. Le système EMIR est un outil de visualisation et de traitement d'image conçu pour une utilisation interactive, et destiné à équiper les Centres Météorologiques Régionaux. Quatre consoles de ce type sont en cours d'installation dont une à Paris. EMIR sera décrit, en détail, au chapitre suivant.

4) Pendant une année au moins, en attendant que le système COMETE décrit plus haut soit opérationnel, une "boite de transfert" dirigera les données arrivant sur Paris vers le Centre de Recherches en Physique de l'Atmosphère de Magny-les-Hameaux, près de Paris, où elles seront enregistrées. Ceci permettra une mise au point plus rapide des méthodes de traitement opérationnel des données radar et satellite.

Le schéma de la figure 4, proposé pour le début de la réalisation du projet, a l'avantage de regrouper les données satellite et radar avec les autres données météorologiques sur un même support informatique, les calculateurs CDC. Un certain contrôle opérationnel des images sera assuré par le service actuel de prévision à courte échéance, ce qui permettra aux météorologistes de se familiariser avec ce nouveau type de données.

Cependant, la première étude conduite à la Météorologie sur la prévision à courte échéance des échos radar montre bien que pendant un certain temps au moins il sera nécessaire d'employer une procédure interactive, afin de corriger certains défauts qui peuvent apparaitre dans l'imagerie radar (masques, propagation anormale, etc...) et de tenir compte des aspects particuliers de chaque situation (persistance et évolution des échos, non conformité des déplacements, etc...). Ces résultats rejoignent ceux obtenus en Grande-Bretagne par Browning sqq (1982) par exemple. Cette étude a portée sur les données d'un radar, celui de Dammartin en Goëlle, pour lequel on dispose de plusieurs années d'enregistrements. Le travail ayant été fait avec un seul radar qui présentait par ailleurs beaucoup d'échos de sol, l'échéance des prévisions a été limitée à deux heures. Dans le cas contraire, on aurait favorisé les situations où les déplacements sont lents. Par ailleurs, les systèmes convectifs sont rarement extrapolables au-delà de deux heures.

On a pu tester sur plusieurs types de situations météorologiques deux méthodes classiques mais fondamentalement différentes, le suivi du centre de gravité des échos et la corrélation croisée sur l'image radar entière. Plusieurs variantes de cette dernière technique ont été essayées. Les tests ont également porté sur les dimensions de la maille utilisée pour la prévision et la vérification des résultats, ainsi que sur le pas de temps.

La méthode de prévision par suivi des échos a été rapidement éliminée, car dépendant énormément de la

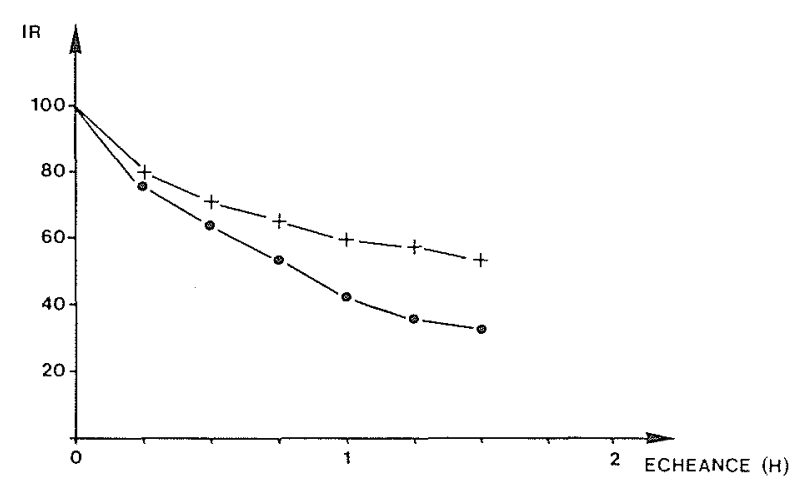

Figure 5 - Comparaison de la qualité des prévisions obtenues par les méthodes de suivi d'échos $\left(0--^{-}\right)$et de corrélation $(+-+)$ pour 15 situations frontales. Les calculs sont faits sur une maille de $4 \times 4 \mathrm{~km}^{2}$ et les indices de Rousseau sont estimés sur une maille de $16 \times 16 \mathrm{~km}^{2}$. 
qualité des données (masques, informations manquantes, effets de distance) et s'appliquant mal aux échos étendus dont le centre de gravité est difficile à déterminer. La corrélation croisée est beaucoup moins sensible à ces deux inconvénients. La figure 5 montre les résultats moyens de la comparaison des deux méthodes, résultats obtenus à partir de 15 prévisions. L'indice de qualité de la prévision utilisé est celui de Rousseau (1980). Cet indice a l'avantage d'être plus sensible que ceux employés habituellement dans la littérature.

Pour la prévision par corrélation croisée on a tout intérêt à conserver une bonne résolution sur les images de départ, surtout dans les situations convectives où les développements d'échos peuvent être très rapides. Par ailleurs, on obtient une meilleure précision des extrapolations. A titre d'exemple, la figure 6 montre des résultats représentatifs, avec des grilles initiales de $4 \times 4,8 \times 8$ et $16 \times 16 \mathrm{~km}^{2}$, toutes les vérifications étant faites sur une grille de $16 \times 16 \mathrm{~km}^{2}$. On a également constaté que les calculs pouvaient être faits sur 1 bit, sans dégradation sensible de la qualité des prévisions. En ce qui concerne le pas dans le temps, il apparait souhaitable pour les situations convectives de réduire l'intervalle entre images à moins de 15 minutes.

La figure 7 donne les taux de bonne prévision et de fausse alerte obtenus sur 15 situations. Ces indices sont définis comme étant le rapport du nombre de

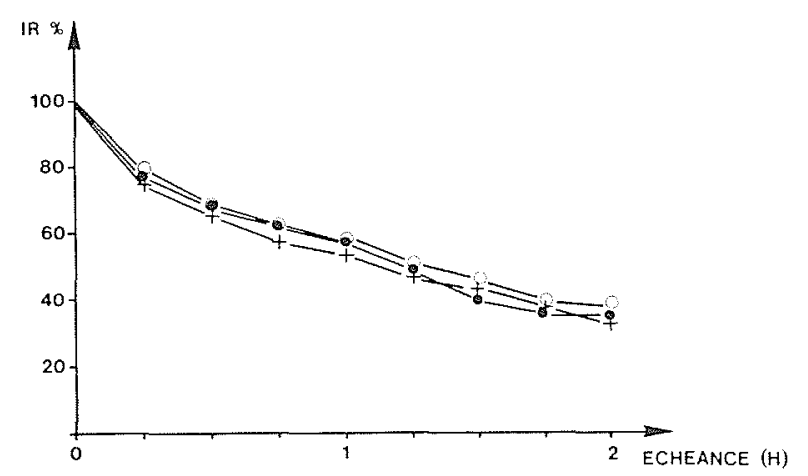

Figure $6 \mathrm{a}$ - Indice de Rousseau en fonction du temps pour des prévisions faites avec des résolutions de départ de $4(0-0)$, $0-08(\%)$ et $16(+-+) \mathrm{km}$, par la méthode de corrélation, Les résultats sont moyennés sur 12 situations frontales, pour des couvertures pluvieuses supérieures à $10 \%$.

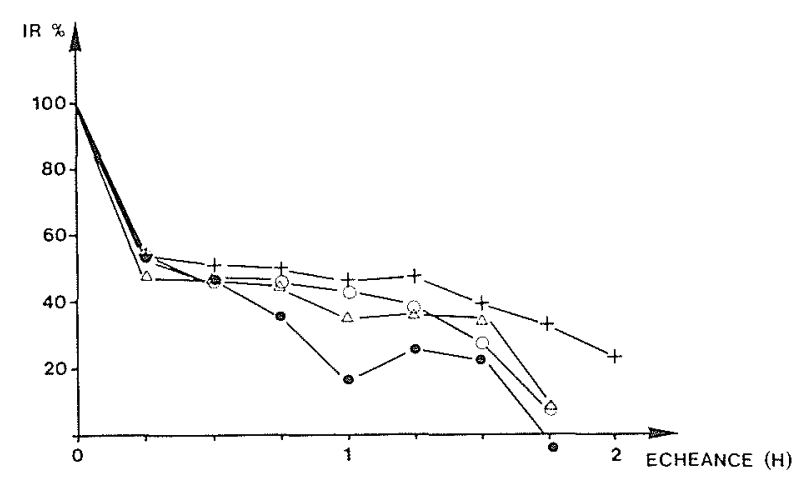

Figure $6 \mathrm{~b}$ - Indice de Rousseau en fonction du temps pour des prévisions faites avec des résolutions de départ de $4(0-0)$, $8\left(0^{\circ}\right)$ è $16(\Delta-\Delta) \mathrm{km}$, par la méthode de corrélation, et $4(+-+) \mathrm{km}$ par corrélation booléenne, pour une ligne de grains dans un tlux de NW rapide ( 7 octobre 1980).

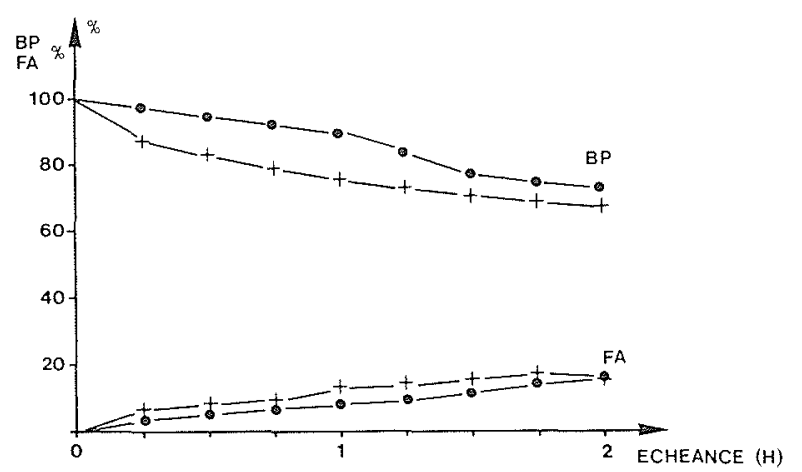

Figure 7 - Taux de fausse alerte (FA) et de bonne prévision (BP) obtenus par la méthode de corrélation $\left(0^{\circ}\right)$ et en admettant la persistance $(x-x)$. La vérification est faite sur une maille de $16 \times 16 \mathrm{~km}^{2}$, en moyennant sur 15 situations frontales.

points correctement prévus au nombre de points total et comme le rapport du nombre de points où il $\mathrm{y}$ a eu fausse alerte au nombre de points total. Ils ne présentent qu'un intérêt très limité pour la comparaison des méthodes de prévision, mais peuvent être très significatifs pour certains usages. En réalité, il nous semble que l'indice, à utiliser pour juger de l'utilité d'une prévision de ce type, dépende très fortement de l'application envisagée, et que les utilisateurs seront amenés à déterminer eux-mêmes l'indice adapté à leurs contraintes économiques.

\section{Diffusion}

La diffusion des données se fera dans un premier temps par lignes spécialisées, soit depuis un radar pour l'image le concernant, soit depuis le service central ou une station alimentée par ailleurs pour les mosaiques radar et les images satellite (Fig. 2). Les images transmises depuis Paris vers les stations météorologiques transiteront par les canaux à $4800 \mathrm{~b} / \mathrm{s}$ utilisés actuellement pour la transmission fac similé des cartes météorologiques. Ce réseau alimente pratiquement toutes les stations départementales de la Météorologie. Parallèlement, les centres météorologiques les plus importants équipés d'une console EMIR recevront également des images satellite en provenance du Centre de Météoro. logie Spatiale de Lannion par une ligne spécialisée à $2400 \mathrm{~b} / \mathrm{s}$.

Il est également prévu de transmettre des données sur réseau commuté, à l'aide de répondeurs placés à Paris, dans les Centres Météorologiques Régionaux ou dans les stations radar. Enfin, pour le grand public une diffusion sur le système TELETEL pourra être envisagée.

La Météorologie a fait industrialiser deux types de stations de réception d'images; ce sont d'une part, le terminal EMIR, adapté à l'usage des Centres Météorologiques Régionaux, d'autre part, le terminal METEOTEL, qui équipera les stations moins importantes.

Le système EMIR, réalisé par SINTRA ALCATEL, peut être considéré comme la juxtaposation de deux éléments.

Le premier élément est un minicalculateur d'usage général avec ses périphériques; le minicalculateur est 
une émulation du MITRA 225 par micro-programmation d'un circuit intégré LSI 2901 à "tranche de bit". Il dispose des périphériques suivants :

- disque 24 Megaoctets

- disquette 1 Megaoctet

- console système

- imprimante ligne

- lignes séries

Le deuxième élément est une console de visualisation couleur avec ses mémoires et ses processeurs vidéo ainsi que la gestion de moyens d'entrée interactifs. La console gère deux mémoires $512 \times 512 \times 8$ bits (extensible à 12) et 1 mémoire alphanumérique de 24 lignes de 96 caractères. La liaison de la console au mini-calculateur est une liaison accès direct mémoire à haute cadence.

Les facilités de traitement des images offertes par le matériel sont très développées car elles permettent toutes transformations de la forme :

$$
h\left[g _ { 1 } \left(f_{1}\left(M_{1}\right)( \pm \mathrm{ET} / \mathrm{OU}) g_{2}\left(f_{2}\left(M_{2}\right)\right] .\right.\right.
$$

où $h, g_{1}, f_{1} g_{2}, f_{2}$ sont des fonctions quelconques exprimées par une table.

Il faut associer à cela la possibilité de recopie du résultat dans une mémoire et donc la possibilité d'expression in téractive.

Le système METEOTEL a été concu avant tout dans un souci d'économie et dans le but d'alimenter un maximum de stations météorologiques. Dans sa version minimale, il est constitué d'un microcalculateur connecté, soit sur le réseau fac similé codé à $4800 \mathrm{~b} / \mathrm{s}$, soit sur une ligne spécialisée aboutissant à un radar. La capacité mémoire correspond à deux images au format METEOTEL, pouvant être des images radar local, des images satellitaires ou des images radar composites. Cer- taines stations météorologiques seront équipées de une ou de deux possibilités supplémentaires de connection sur des lignes téléphoniques, d'une capacité mémoire de 15 images et de la possibilité de sortie sur imprimante et/ou magnétophone. Toutes les versions seront équipées d'un clavier de commande permettant certaines manipulations sur les images.

Les stations METEOTEL sont conçues autour d'un Bus de communication entre les cartes très répandu, le Bus IEEE 796, ce qui permettra de les faire évoluer ultérieurement en fonction des besoins qui ne manqueront pas de se préciser après leur mise en exploitation.

\section{Conclusions}

Le projet ARAMIS constitue une des priorités du Service Météorologique Français. Les efforts demandés pour la constitution du réseau de radars se justifient entièrement par les seuls besoins propres à ce service. Le programme présenté ici consiste, pour l'essentiel, à modifier légèrement les matériels qui existent déjà à la Météorologie et à les interconnecter.

On entrevoit de nombreuses possibilités d'utilisation des données radar par d'autres services, comme l'aéronautique, la protection civile ou les agences de bassin. Plusieurs expériences ont déjà eu lieu dans ce seńs; si elles n'ont pas toutes été positives, elles démontrent que le radar est un instrument qui, comme tous les autres, a ses limites, bien qu'il puisse être adapté à beaucoup d'usages. Nous estimons que malgré les nombreuses difficultés qui pourront encore se présenter, les utilisations opérationnelles du radar pour la détection des phénomènes dangereux, la prévision à très courte échéance et la mesure hydrologique, ont un bel avenir.

\section{Bibliographie}

BROWNING K.A., COLLIER C.G., LARKE P.R., MENMUIR P., MONK G.A. et OWENS R.G. - On the forecasting of frontal rain using a weather radar network. Mon. Wea. Rec., $1982,110,534-552$.

FROMENT G. - L'expérience HYDROMEL. Rapport technique final. 1978, Etablissement d'Etudes et de Recherches Météorologiques, 77 , rue de Sèvres, 92 Boulogne.

ROUSSEAU D. - $A$ new skill score for the evaluation of yes/no forecasts. WMO Symposium on probabilistic and statistical methods in weather forecasting, Nice, Sep. 1980, pp. 167-174.

WILSON J.W. and BRANDES E.A. - Radar Measurements of rainfall - a summary - Bull Amer. Meteor. Soc., 1979, 60, 1048-1058. 


\section{Discussion}

M. GUILLOT. - Pour mieux valoriser des sites encaissées comme Lyon et Marseille, est-ce que vous n'auriez pas intérêt à déplacer ces radars sur une éminence?

M. GILET. - Ce serait certainement la solution la plus logique. Sans revenir sur les raisons qui ont conduit à installer les radars là où ils sont, c'est un fait que certains sont installés dans des sites qui ne conviennent pas parfaitement.

Mais pour répondre de façon plus précise à votre question, il n'y a pas actuellement de décision prise de déplacer les radars de Lyon et de Marseille.

M. CHOCAT. - Pouvez-vous apporter des précisions sur les modalités pratiques de la mise à disposition des images radar à des usagers extérieurs à la Météorologie Nationale?

M. GILET. - Pour certains radars comme celui de Dammartin, il n'y a pas de difficultés techniques pour qu'un utilisateur extérieur reçoive des images. Mais il n'y a pas actuellement de politique systématique. Il suffit, comme cela a été fait pour la D.D.E. de Seine Saint-Denis d'utiliser une station de réception SAPHYR et de la brancher sur une ligne téléphonique spécialisée.

Plus tard, il sera possible d'acheter des stations METEOTEL plus performantes et de récupérer différentes images par l'intermédiaire d'une ligne spécialisée, ou directement par le réseau téléphonique commuté.

Enfin pour la station de Dammartin, on commence à faire des expériences sur TELETEL.

M. LE QUENTREC. - Je précise que pour TELETEL, ce sont plutôt des images simplifiées qui sont transmises.

M. HORRIE. - Pour le projet ARAMIS, vous avez parlé d'un rayon d'action de $250 \mathrm{~km}$ pour les différents radars. Est-ce que l'on ne vise pas un peu trop haut à cette distance là !

M. GILET. - $250 \mathrm{~km}$, c'est le rayon de l'image qu'on transmet. A cette distance, lorsqu'on regarde des orages, on voit surtout le sommet des nuages et l'image ne peut donc pas être interprétée de manière quantitative. Dans le cas de systèmes frontaux d'hiver, on les détecte rarement à plus de $200 \mathrm{~km}$. II demeure cependant intéressant, même à cette distance, de détecter ce qu'on peut détecter.

M. HORRIE. - Comment prévoyez-vous de résoudre le problème de l'étalonnage des radars?

M. GILET. - La politique actuelle de la Météorologie Nationale est de décentraliser au maximum la maintenance et l'exploitation des radars. Mais c'est un point sensible, car l'équipement des radars n'est peut-être pas suffisant aujourd'hui pour pouvoir réaliser des étalonnages très précis. Sans doute y aura-t-il quelques problèmes au départ, mais je pense que cela devrait se régler progressivement.

M. $B A C H O C$. - Vous avez dit que c'était pour vous un peu une deuxième urgence; mais est-ce que les utilisateurs qui souhaiteraient calibrer eux-mêmes des images radar pourraient, malgré tout, compter sur un appui de la Météorologie Nationale ?

M. GILET. - Comme l'a expliqué M. SAUVAGEOT, la meilleure méthode d'étalonnage consiste à ajuster les données radar sur des données pluviographiques. C'est une opération que nous n'avons pas placée dans la phase initiale du programme ARAMIS, car il a fallu effectuer des choix, notre budget étant limité. Cela suppose en effet une informatisation plus poussée du radar, et passe peut-être aussi par l'automatisation de la collecte des données pluviographiques, opérations pouvant être très onéreuses.

Mais si des utilisateurs extérieurs veulent réaliser ce type d'étalonnage, je pense que la Météorologie pourra apporter son soutien au niveau des méthodes.

M. AUCHET. - Quel est le délai de mise à disposition d'une image radar?

M. GILET. - Si la transmission se fait directement à partir du radar, il est d'une à deux minutes. Par contre, dans le cas des images centralisées, le délai est plus important, car les traitements à effectuer sont plus nombreux. Je pense qu'il sera de l'ordre du quart d'heure, peut-être même un peu plus. Mais cela reste à voir en pratique.

M. PARSY. - Quel est le nombre de caractères à transmettre pour une image complète, et pourquoi ne pas utiliser le réseau TRANSPAC qui permet de travailler à 4800 bauds?

M. GILET. - Le nombre de données à transmettre est de 32000 octets, ce qui demande environ 7 à 8 minutes de transmission sur une ligne à 600 bauds. La solution TRANSPAC, elle, n'a pas encore été vraiment envisagée; mais effectivement cela permettrait des transmissions plus rapides, de l'ordre de la minute si l'on travaillait à 4800 bauds.

MM. P. DAVID et P. BISSONNIER, du Centre technique et du matériel de la Météorologie, ont présenté en fin de séance un film montrant la dynamique de systèmes précipitants dans le cas de quatre stations météorologiques différentes.

M. GUILLOT. - Pour les situations que vous nous avez montrées, l'une en été notamment et l'autre en hiver, il semble que l'on observe des échos plus lointains dans un cas que dans l'autre. Est-ce à dire qu'en été on peut voir jusqu'à $300 \mathrm{~km}$, mais qu'en hiver il faut se limiter à $200-250 \mathrm{~km}$ ?

M. DA VID. - C'est un peu cela. Lors d'orages violents, on réussit à avoir une détection jusqu'à $300 \mathrm{kms}$, ce qui est une sorte d'indice de la sévérité du phénomène, car cette distance est liée à l'extension verticale des nuages. En hiver, par contre, sur des lignes frontales de faible extension verticale, on ne peut pas dépasser 150 à $200 \mathrm{~km}$, car au-delà le faisceau est trop haut.

M. GUILLOT. - Mais si l'on ne détecte rien, est-ce parce que l'on est trop haut ou parce que l'on est dans la neige?

M. $D A V I D$. - C'est parce qu'il n'y a rien ou qu'il n'y a que des cristaux très fins. 


\section{Abstract}

The Aramis project and short-range forecasting

In order to improve knowledge in real time of the three-dimensional distribution of precipitation and to forecast rain more accurately within a range of a few hours the Meteorological office has set up a radar network which will make it possible to plot precipitation every fifteen minutes with an accuracy of several kilometers over the major part of France. This network consists of 10 radars with 5 or $10 \mathrm{~cm}$ wavelengths.

Each radar is provided with a digital system which controls the aerial acquisition and processing of data and telephonic transmission of the resultant pictures. A processing centre in
Paris will receive the pictures from all the radars and establish composite precipitation charts at national level. The data will be analysed in relation to satellite information and other meteorological data available at the central data bank. Great efforts will be made to promote extremely rapid dissemination of the pictures to all meteorological staions and other interested users. The latter will be able to install METEOTEL picture reception cabinets, so that they can receive pictures direct from a given rada or the composite picture established in paris. 